\title{
Validation of finite element solutions of nonlinear, periodic eddy current problems
}

\author{
RenÉ Plasser, OSZKÁR Bíró \\ Institute of Fundamentals and Theory in Electrical Engineering \\ Graz University of Technology, Inffeldgasse 18, 8010 Graz, Austria \\ e-mail: rene.plasser@tugraz.at
}

(Received: 14.07.2014, revised: 17.09.2014)

\begin{abstract}
An industrial application is presented to validate a finite element analysis of 3-dimensional, nonlinear eddy-current problems with periodic excitation. The harmonicbalance method and the fixed-point technique are applied to get the steady state solution using the finite element method. The losses occurring in steel reinforcements underneath a reactor due to induced eddy-currents are computed and compared to measurements.
\end{abstract}

Key words: eddy-currents, nonlinear magnetics, finite elements, 3-dimensional

\section{Introduction}

The focus of this investigation is to determine the additional losses of a dry insulated air reactor. There is no iron core or insulation fluid present in such reactors. Air reactors are used in different sections of electrical industry for example in distribution networks to compensate reactive power or capacitive currents in long low powered distribution lines, short circuit limiting reactors and also in measurement setups. In any way they are used, such air reactors can reach huge dimensions, and one has to guarantee that the seating of the reactor is capable to withstand its weight. To ensure this, it is reinforced with a steel grid.

Due to the time-varying magnetic field of the reactor, eddy currents are induced in the conducting, highly permeable grid in the vicinity of the reactor.

These eddy-currents cause additional losses. One has to deal with a nonlinear problem because of the ferromagnetic behaviour of the grid material. In this case the excitation is timeperiodic and the steady state periodic solution is of interest [1]. Such problems can be solved in the time domain using the time-periodic finite element method [2-4]. In this work, we have chosen a frequency domain approach. At first the problem has been solved using the time-harmonic approximation. For further investigations, the fixed point technique has been employed to take account of higher harmonics. The fixed point technique has been applied to the harmonic balance method to decouple the harmonics and to linearize the system of algebraic equations for each iteration step by selecting a time-independent permeability distribution. This 
yields the advantage that each harmonic can be obtained by solving an equation system with as many unknowns as there are finite element degrees of freedom. Since these systems of algebraic equations are independent of each other they can be solved parallel which is a great benefit in saving computational time. The finite element method (FEM) has been applied to the so called $\mathbf{A}_{r}, V-\mathbf{A}_{r}$ reduced vector potential formulation [5].

\section{FEM-formulation and modeling}

To solve the problem with the aid of the finite element method, one has to distinguish between two geometrical domains: an eddy-current domain with an unknown eddy-current density and an eddy-current free region, in which the current density is given. In this application, the domain of the known current density is the coil of the reactor. In the whole region including the conducting and non-conducting areas one can write

$$
\mathbf{B}=\mu_{0} \mathbf{H}_{s}+\operatorname{curl} \mathbf{A}_{r} .
$$

Here $\mathbf{B}$ is the magnetic flux density, $\mathbf{H}_{s}$ is the impressed magnetic field intensity given by applying Biot-Savart's law to the impressed current density of the coil $\mathbf{J}_{0} . \mathbf{A}_{r}$ is the reduced magnetic vector potential and $\mu_{0}$ is the vacuum permeability.

For the conducting domain, we have [5]:

$$
\mathbf{E}=-\frac{\partial}{\partial t} \mathbf{A}_{r}-\frac{\partial}{\partial t} \operatorname{grad} V-\frac{\partial}{\partial t} \mathbf{A}_{s},
$$

where $\mathbf{E}$ is the electric field intensity, $V$ is an electric scalar potential, and $\mathbf{A}_{s}$ is the impressed vector potential satisfying

$$
\operatorname{curl} \mathbf{A}_{s}=\mu_{0} \mathbf{H}_{s} \text { and } \operatorname{curl} \mathbf{H}_{s}=\mathbf{J}_{0} .
$$

Considering the material relations $\mathbf{H}=v \mathbf{B}$ and $\mathbf{J}=\sigma \mathbf{E}$ where $v$ is the magnetic reluctivity and $\sigma$ is the electrical conductivity and using (1), (2) and (3), Maxwell's equations lead to [6-8]:

$$
\begin{aligned}
\operatorname{curl}\left(v \operatorname{curl} \mathbf{A}_{r}\right)+\sigma \frac{\partial}{\partial t} \mathbf{A}_{r}+\sigma \frac{\partial}{\partial t} \operatorname{grad} V & =-\sigma \frac{\partial}{\partial t} \mathbf{A}_{s}-\operatorname{curl}\left(v \mu_{0} \mathbf{H}_{s}\right), \\
-\operatorname{div}\left(\sigma \frac{\partial}{\partial t} \mathbf{A}_{r}+\sigma \frac{\partial}{\partial t} \operatorname{grad} V\right) & =\operatorname{div}\left(\sigma \frac{\partial}{\partial t} \mathbf{A}_{s}\right) .
\end{aligned}
$$

Applying Galerkin's method to the differential Equations (4) and (5), one obtains an algebraic equation system of ordinary differential equations

$$
\mathbf{S}[v(\mathbf{x}(t))] \mathbf{x}(t)+\mathbf{M}(\sigma) \frac{d \mathbf{x}(t)}{d t}=\mathbf{f}(t),
$$

where $\mathbf{S}$ represents the stiffness matrix depending on $v$. The matrix $\mathbf{M}$ is the mass matrix depending on the electrical conductivity $\sigma$. The vector $\mathbf{x}$ gathers the unknown vector and scalar 
potentials and depends on the time $t$. The reluctivity is varying with the magnetic field and depends on $\mathbf{x}$ and hence on $t$. On the right hand side of the equation the vector $\mathbf{f}$ represents the given quantities.

\subsection{The time-harmonic approximation}

As a first approach, the time-harmonic approximation [9] has been used by transforming the differential Equation (6) in the frequency domain under the assumption of sinusoidal time variation. Since the derivative in the time domain corresponds to a multiplication by $j \omega$, one obtains a complex, nonlinear equation system in the frequency domain of the form

$$
[\mathbf{S}(v)+j \omega \mathbf{M}(\sigma)] \mathbf{X}=\mathbf{F} .
$$

In this equation $j$ is the imaginary unit and $\omega$ is the angular frequency. The vector $\mathbf{X}$ gathers the complex amplitudes of the unknown vector and scalar potentials. On the right hand side of the equation we have the vector $\mathbf{F}$ depending on the given quantities. This equation system has to be modified if higher harmonics are to be considered.

\subsection{Higher harmonics using the fixed-point method}

One can consider higher harmonics too, by using a complex Fourier-series to approximate the potentials [10], [11]. Then, $\omega$ in Equation (7) takes on several discrete values leading to a harmonic balance technique:

$$
\mathfrak{F}_{m}\{\mathbf{S}[v(\mathbf{x})] \mathbf{x}\}+j m \omega \mathbf{M}(\sigma) \mathbf{X}_{m}=\mathfrak{F}_{m}(\mathbf{f}),
$$

where $m=1,2, \ldots, N$ is the order of the harmonics and $\mathfrak{F}_{m}(\mathbf{f})$ represents the $m$-th harmonic in the complex Fourier transform of the time domain right hand side $\mathbf{f}$ which is computed directly (see (16) below). If the problem is linear, these equations are independent for each harmonic, but, due to nonlinearity, i.e. to the dependence of the reluctivity $v(\mathbf{x})$ on $\mathbf{x}$, they are all coupled, leading to an equation system with $N$ times as many unknowns as there are degrees of freedom in (7).

To decouple the equations in the nonlinear case as well, the fixed-point iteration method $[7,12,13]$ is used to solve $(8)$.

This method is capable to find the fixed point of a nonlinear function. Generally the fixed point $\mathbf{x}_{F P}$ of the function $\mathbf{I}\left(\mathbf{x}_{F P}\right)$ is defined as

$$
\mathbf{x}_{F P}=\mathbf{I}\left(\mathbf{x}_{F P}\right) .
$$

The solution is found as the intersection of two functions $\mathbf{y}=\mathbf{I}\left(\mathbf{x}_{F P}\right)$ and $\mathbf{y}=\mathbf{x}_{F P \text {. A series }}$ of the form

$$
\mathbf{x}^{(s+1)}=\mathbf{I}\left(\mathbf{x}^{(s)}\right),
$$

where the superscript in brackets $s=0,1,2, \ldots$ indicates the iteration count, converges to the fixed point $\mathbf{x}_{F P}=\mathbf{I}\left(\mathbf{x}_{F P}\right)$, if the mapping corresponding to the function $\mathbf{I}(\mathbf{x})$ is a contraction, i.e. there exists a constant $q<1$ so that for any $\mathbf{x}_{1}$ and $\mathbf{x}_{2}$ the inequality 


$$
\left\|\mathbf{I}\left(\mathbf{x}_{1}\right)-\mathbf{I}\left(\mathbf{x}_{2}\right)\right\| \leq q\left\|\mathbf{x}_{1}-\mathbf{x}_{2}\right\|
$$

holds.

A general nonlinear equation $\mathbf{F}(\mathbf{x})=0$ can be transformed to a fixed point problem by selecting a suitable linear operator $\mathbf{A}$ and defining $\mathbf{I}$ as

$$
\mathbf{I}(\mathbf{x})=\mathbf{x}+\mathbf{A}^{-1} \mathbf{F}(\mathbf{x}) .
$$

The iterations of (10) then become

$$
\mathbf{A}^{(s)} \mathbf{x}^{(s+1)}=\mathbf{A}^{(s)} \mathbf{x}^{(s)}+\mathbf{F}\left(\mathbf{x}^{(s)}\right),
$$

where the superscript of the linear operator $\mathbf{A}$ indicates that it can be changed at each iteration step to achieve faster convergence.

A simple example for using the fixed-point method in the context of magnetic nonlinearity is shown in Figure 1.

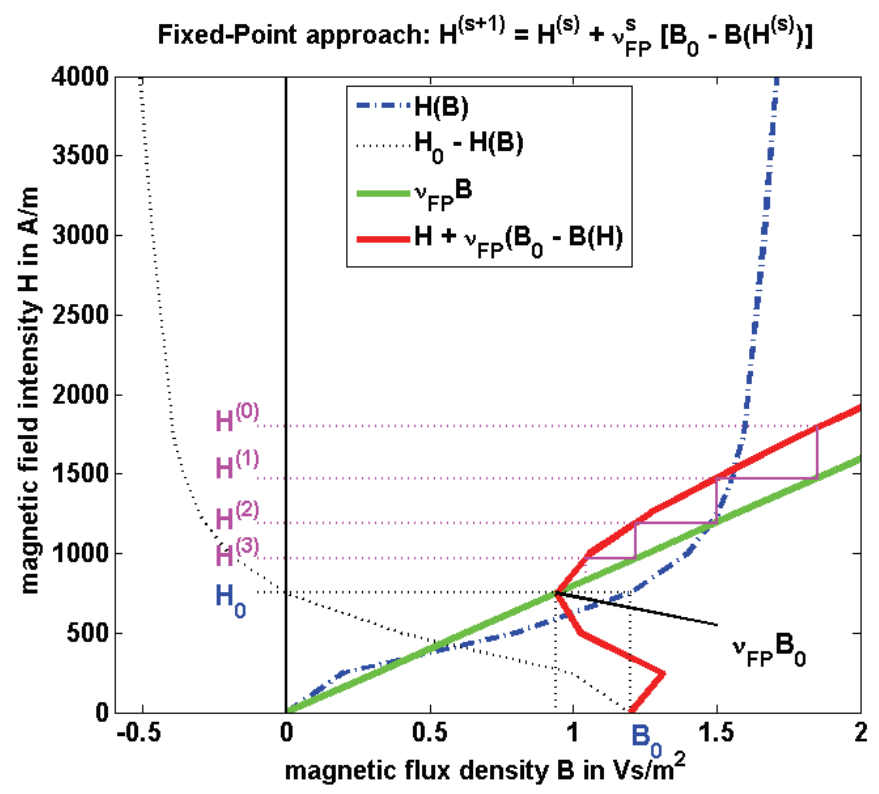

Fig. 1. Fixed-point iteration steps with a constant value of the magnetic fixed point reluctivity $v_{\mathrm{FP}}$

For the example in Figure 1 (13) leads to

$$
\mathbf{H}^{(s+1)}=\mathbf{H}^{(s)}+\boldsymbol{v}_{F P}^{(s)}\left(\mathbf{B}_{0}-\mathbf{B}\left(\mathbf{H}^{(s)}\right)\right) .
$$

As mentioned before, convergence is guaranteed for contractive problems. This is given if the fixed-point reluctivity is chosen as in [13]. The $\mathbf{H}(\mathbf{B})$ curve can be given as a piece-wise linear monotonous function. The fixed-point technique will converge for any starting value and the system matrix does not need to be updated in every iteration step. Only the right-hand 
side of the equation system needs to be adjusted. Due to the linearized problem in each iteration step and the independence of the equations of the decoupled harmonics, these can be calculated in parallel, a feature leading to substantial saving of computational time.

The value of the magnetic reluctivity $v_{\mathrm{FP}}$ is not dependent on the field and hence time, but may vary with the space coordinates of the problem domain. Therefore, this parameter can be changed in each iteration step. In the problem shown the magnetic permeability $v_{F P}$ is assumed to stay constant. To get optimal convergence the best way to vary the fixed-point permeability $\mu_{F P}$ or reluctivity $v_{\mathrm{FP}}$ is explained in [13].

Since the nonlinearity in (8) is due to the dependence of $v(\mathbf{x})$ on the solution, it is obvious to define a field independent fixed-point reluctivity $v_{\mathrm{FP}}$.

Hence, one has to solve the following equation system in each iteration step $s=1,2, \ldots$ :

$$
\left[\mathbf{S}\left(v_{F P}^{(s)}\right)+j m \omega \mathbf{M}(\sigma)\right] \mathbf{X}_{m}^{(s+1)}=\mathfrak{F}_{m}\left(\mathbf{f}+\mathbf{S}\left(v_{F P}^{(s)}-v^{(s)}\right) \mathbf{x}^{(s)}\right),
$$

where $v_{F P}^{(s)}$ is the fixed point reluctivity at the $s$-th iteration step and $\mathfrak{F}_{m}(\mathbf{x})$ denotes the $m$-th harmonic of the Fourier transform defined as

$$
\mathfrak{F}_{m}(\mathbf{x})=\frac{1}{T} \int_{0}^{T} \mathbf{x} e^{-j \omega m t} d t .
$$

The time domain solution $\mathbf{x}$ in the $(s+1)$-th iteration step can be obtained by inverse Fourier transform using

$$
\mathbf{x}^{(s+1)}=\mathfrak{F}^{-1}\left(\mathbf{X}_{m}^{(s+1)}\right),
$$

where

$$
\mathfrak{F}^{-1}\left(\mathbf{X}_{m}\right)=\sum_{m=-\infty}^{\infty} \mathbf{X}_{m} e^{-j \omega m t}
$$

\section{Numerical investigations}

To validate the method, results calculated from the finite element solution of a specific nonlinear periodic eddy-current problem have been compared to measured values. The losses in the steel reinforcements underneath an air reactor have been investigated (see Fig. 2). To get the additional losses occurring in the steel-grid, the measurement of the losses was first carried out without the steel grid underneath the reactor. In a second step, the coil was placed on the grid and the losses were measured again. The difference between the two values represents the additional loss caused by the induced eddy-currents in the highly permeable steel grid. The used grid is a commercial standard for concrete reinforcements. The steel bars have a round cross-section with a diameter of $8 \mathrm{~mm}$. The mesh width of the grid is $300 \mathrm{~mm}$ by $300 \mathrm{~mm}$ and the whole structure spans an area of $4500 \mathrm{~mm}$ by $4500 \mathrm{~mm}$. All bar intersections 
are welded together. The dimensions of the model are based on the given test setup. The FEM model of the domain is shown in Figure 3. The main parameters of the air reactor are given in Table 1.

Table 1. Main data of the air reactor used in the test setup

\begin{tabular}{l|c|c|c}
\hline Active winding height & $3133 \mathrm{~mm}$ & Rated voltage & $34.5 \mathrm{kV}$ \\
\hline Average winding diameter & $2803 \mathrm{~mm}$ & Rated power & $28.028 \mathrm{MVar}$ \\
\hline Radial winding width & $249 \mathrm{~mm}$ & Rated current & $1015 \mathrm{~A}$ \\
\hline Number of windings & 231.375 & Total mass & $6850 \mathrm{~kg}$ \\
\hline
\end{tabular}

In Figure 2 indicated as a) and coulored red in Figure 3 is the coil of the reactor. Due to the used FEM-formulation the coil is energized with the impressed current densitiy $\mathbf{J}_{0}$. For the finite element analysis, a quarter model is sufficient, because the arrangement is symmetrical. The area of interest is marked as c) and indicated green and shows the steel grid where the eddy-currents are induced. Figure 3 shows the whole domain including the grid of divisions and subdivisions for the macro elements and finite elements marked $b$ ).

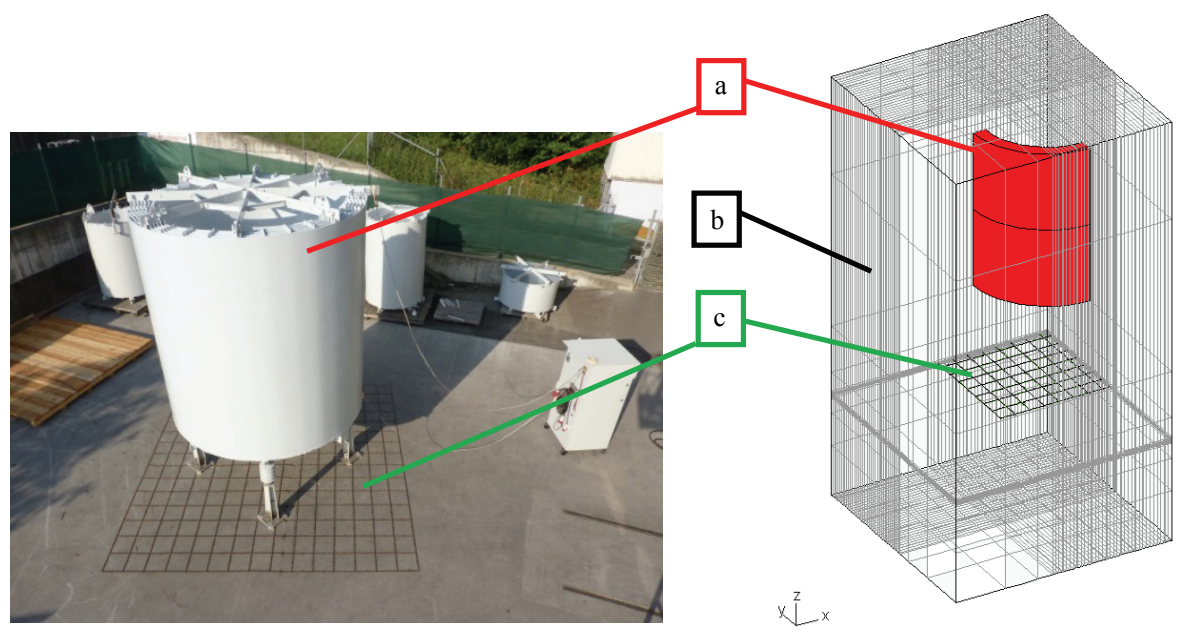

Fig. 2. Test setup

Fig. 3. Quarter-model of the problem

In the section of induced eddy-currents one has to be as close to the real model as possible to get accurate results. As one can see in the close up in Figure 4, the steel bars have a round cross section except at the welding point where the model has the shape of a cone modelling the intersection. The nonlinearity of the material is considered by the implementation of the B-H curve of the material given by the manufacturer.

The field- and the loss-calculations have been carried out first using the time harmonic approximation and then the harmonic balance approach with up to 11 harmonics solved by the fixed-point method. 


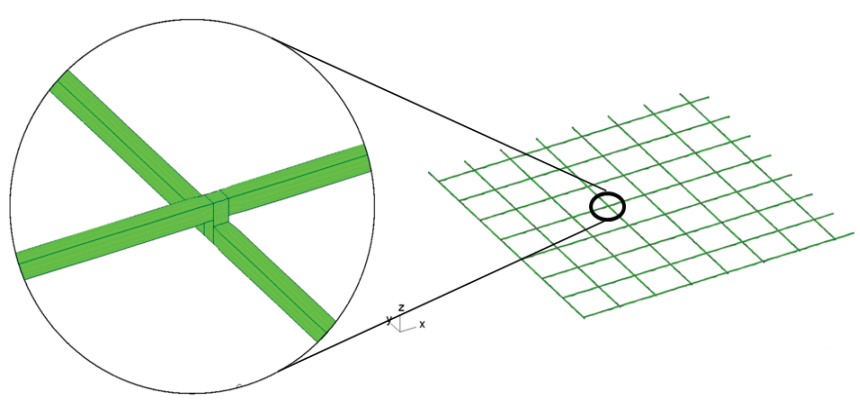

Fig. 4. Close up of the steel grid model

Afterwards, the eddy-current loss calculation was done by evaluating a volume integral over the $\mathbf{A}_{\text {red }}-V$ domain and averaging one time period:

$$
P_{e d d y}=\frac{1}{T} \int_{0}^{T} \int_{\Omega_{c}} \frac{|\mathbf{J}|^{2}}{\sigma} d \Omega d t
$$

In (19), $P_{\text {eddy }}$ indicates the additional losses caused by the induced eddy-currents, $T$ stands for one time period, $\mathbf{J}$ is the current density caused by the eddy-currents and $\Omega_{c}$ marks the volume of interest where the eddy-currents occur.

Table 2. Comparison of measured and calculated eddy-current losses

\begin{tabular}{c|c|c|c|c}
\hline $\mathbf{N r}$ & $\boldsymbol{I}$ & $\boldsymbol{P}_{\boldsymbol{m}}$ & $\boldsymbol{P}_{\boldsymbol{t h}}$ & $\boldsymbol{P}_{\boldsymbol{F} \boldsymbol{P}}$ \\
\hline & $\mathrm{A}$ & $\mathrm{kW}$ & $\mathrm{kW}$ & $\mathrm{kW}$ \\
\hline 1 & 50 & 0.074 & 0.046 & 0.048 \\
2 & 100 & 0.272 & 0.185 & 0.183 \\
3 & 300 & 2.777 & 2.535 & 2.376 \\
4 & 500 & 10.106 & 9.385 & 8.693 \\
5 & 700 & 20.158 & 22.468 & 20.538 \\
6 & 1000 & 43.364 & 54.201 & 49.868 \\
\hline
\end{tabular}

Table 2 shows the measured and calculated losses for different values of reactor current. In Table $2 I$ is the current of the reactor, $P_{m}$ indicates the measured additional losses, $P_{t h}$ lists the eddy-current losses using the time harmonic approach and $P_{F P}$ indicates the computed losses with the fixed-point method considering up to 11 harmonics.

The results plotted in Table 2 are shown graphically in Figure 5.

Using the time-harmonic approximation, the calculated losses differ considerably from the measured ones when the winding current rises. This occurs because the saturation of the steel grid is more significant for higher currents. Taking account of higher harmonics, the deviation is less pronounced. 


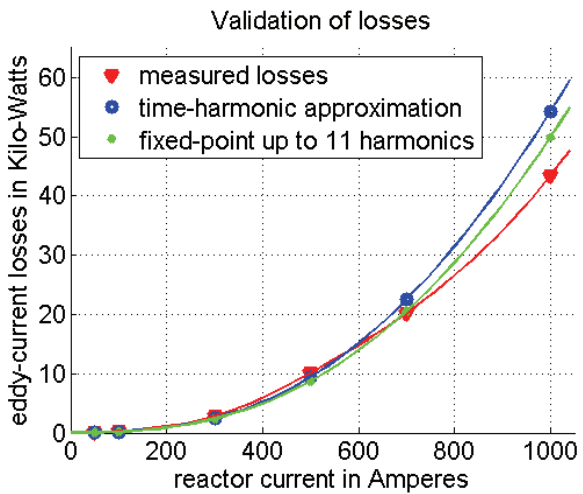

Fig. 5. Validation of losses

To see how expensive the consideration of higher harmonics is, the computational effort is summarized in Table 3 for different current values.

Table 3. Computational information

\begin{tabular}{l|c|c|c|c|c|c|c}
\hline & & \multicolumn{4}{|c}{ Time harmonic } & \multicolumn{3}{c}{$\begin{array}{c}\text { Fixed-point up to 11 } \\
\text { harmonics }\end{array}$} \\
\hline Current & A & 50 & 500 & 1000 & 50 & 500 & 1000 \\
\hline Approx. RAM & GB & \multicolumn{6}{|c}{25} \\
\hline CPU & & \multicolumn{6}{|c}{$2 \times$ Intel Xeon E5-2630 $(6 \times 2.00 \mathrm{GHz}-12$ cores $)$} \\
\hline Nr of equations & & \multicolumn{7}{|c|}{$2,078,464$} & \\
\hline Computational time & $\mathrm{h}$ & 1.5 & 3.5 & 5.5 & 19.5 & 67 & 105.5 \\
\hline
\end{tabular}

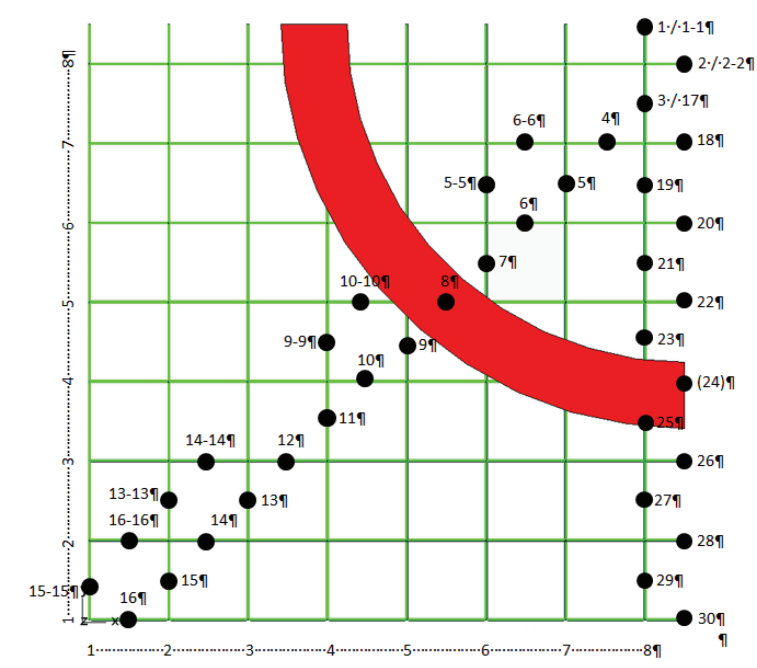

Fig. 6. Shows the points were the current in the grid was measured 


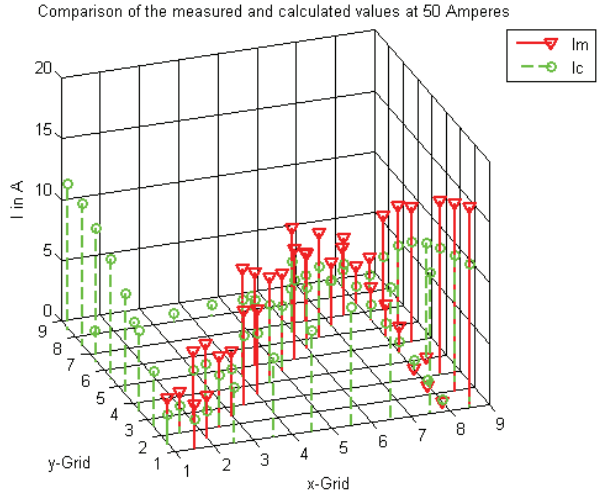

Fig. 7. Current distribution@ 50 A

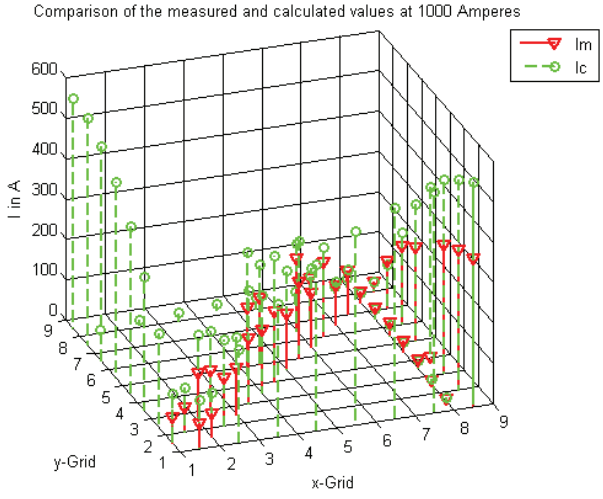

Fig. 8. Current distribution@1000 A

In Figures 7 and 8 one can see the currents in the grid segments marked in Figure 6. The calculated currents are indicated green and denoted by $I_{c}$. The red plots are the measured currents denoted by $I_{m}$. The current values are root mean square values.

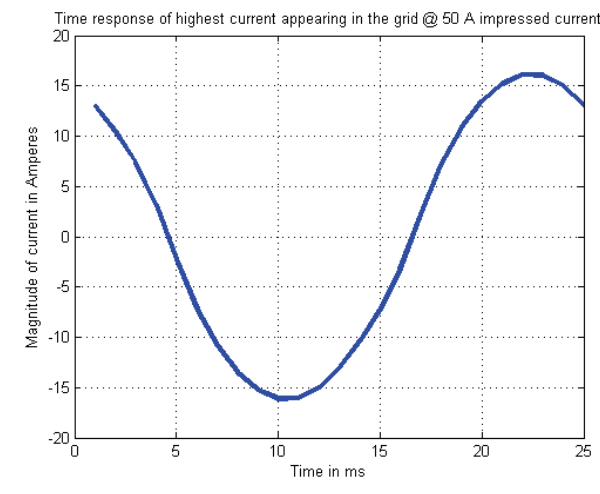

Fig. 9. Eddy-current time response @ 50 A impressed current

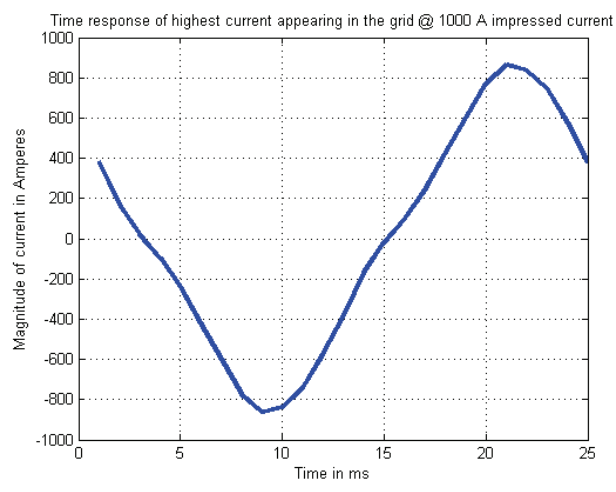

Fig. 10. Eddy-current time response @ 1000 A impressed current

The figures below show the time response of the induced currents in the grid segment 30 marked in Figure 6 when the coil of the reactor is energized with 50 A (Fig. 9) and with 1000 A (Fig. 10). One can clearly see the deformation of the current response due to the nonlinearity when the grid gets saturated at higher current levels.

\section{Conclusion}

For higher currents, the time-harmonic method is not acceptable. The accuracy of the model using 11 harmonics is still not sufficient. Further calculations have to be carried out to mi- 
nimize the deviation between the measured and calculated losses by increasing the number of harmonics taken into account. Also other parameters have to be considered in the calculation like the conductivity decreasing with higher temperature which is of course a major factor because the steel bars of the grid can reach very high temperatures due to the induced eddy-currents.

\section{Acknowledgement}

The authors would like to thank the TRENCH Austria $\mathrm{GmbH}$ for providing us with the measurements and their experience in reactor design.

\section{References}

[1] Paoli G., Buchgraber G., Bíró O., Complex Representation in Nonlinear Time Harmonic Eddy Current Problems. IEEE Trans. on Magn. 34(5): 2625-2628 (1998).

[2] Takahashi Y., Tokumasu T., Kameari A. et al., Convergence acceleration of time-periodic electromagnetic field analysis by the singularity decomposition-explicit error correction method. IEEE Trans. on Magn. 46(8): 2947-2950 (2010).

[3] Takahashi Y., Iwashita T., Nakashima H. et al., Parallel time-periodic finite-element method for steady-state analysis of rotating machines. IEEE Trans on. Magn. 48(2): 1019-1022 (2012).

[4] Takahashi Y., Tokumasu T., Fujita M. et al., Comparison Between Fast Steady-State Analysis Methods for Time Periodic Nonlinear Magnetic Field Problems. IEEE Trans. On Magn. 48(2): 235-238 (2012).

[5] Bíró O., Edge element formulations of eddy current problems. Comput. Methods Appl. Mech. Eng. 169: 391-405 (1999).

[6] Bíró O., Preis K., An Edge Finite Element Eddy current Formulation Using a Reduced Magnetic and a Current Vector Potential. IEEE Trans. on Magn. 36(5): 3128-3130 (2000).

[7] Ciric I.R., An Efficient Harmonic Method for Solving Nonlinear Time-Periodic Eddy-Current Problems. IEEE Trans. on Magn. 43(4): 1185-1188 (2007).

[8] Hara T., Naito T., Umoto J., Time-Periodic Finite Element Method for Nonlinear Diffusion Equations. IEEE Trans. on Magn. 21(6): 2261-2264 (1985).

[9] Albanese R., Coccorese E., Martone R. et al., Periodic Solutions of Nonlinear Eddy Current Problems in Three-Dimensional Geometries. IEEE Trans. on Magn. 28(2): 1118-1121 (1992).

[10] Ausserhofer S., Bíró O., Preis K., An efficient Harmonic Balance Method for nonlinear eddy current problems. IEEE Trans. on Magn. 43(4): 1229-1232 (2007).

[11] Weymann J., Thomas P., Gaombalet J., A New Method for Periodic Solutions of Nonlinear Eddy Current Problems. IEEE Trans. on Magn. 35(3): 1115-1118 (1999).

[12] Matsuo T., Time-Periodic Finite Element Method for Hysteretic Eddy-Current Analysis. IEEE Trans. on Magn. 38(2): 549-552 (2002).

[13] Koczka G., Ausserhofer S., Bíró O., Preis K., Optimal convergence of the Fixed-Point Method for Nonlinear Eddy Current Problems. IEEE Trans. on Magn. 45(3): 948-951 (2000). 